\title{
Immersive Live Sports Experience with Vibrotactile Sensation
}

\author{
Beom-Chan Lee, Junhun Lee, Jongeun Cha, Changhoon Seo, and Jeha Ryu \\ Human-Machine-Computer Interface Lab., \\ Dept. Mechatronics, Gwangju Institute of Science and Technology, Gwangju, Korea \\ \{bclee, junhun, gaecha, search, ryu\}@gist.ac.kr
}

\begin{abstract}
This paper presents a vibrotactile display system designed with an aim of providing immersive live sports experience. Preliminary user studies showed that with this display subjects were $35 \%$ more accurate in interpreting an ambiguous visual stimulus showing a ball either entering or narrowly missing a football net. About $80 \%$ of subjects could judge the correct ball paths in the presences of ambiguous visual stimuli. Without the tactile display, only $60 \%$ correct paths are judged from the visual display.
\end{abstract}

\section{Introduction}

The rapid development of broadcasting technology has made it possible to provide TV viewers with high quality visual and auditory services yielding a more realistic and impressive experience of digital content. However, we believe, there exists futher potential. Specifically we are concerned with the possibilities afforded by the additional of haptic information to a broadcast stream [1], [2].

In TV broadcasting of live sports, sometimes confusing situations occur. For example, when a soccer ball passes close to the edge of a goalpost, viewers may be unable to determine whether or not a goal was scored because the visual scene gives only $2 \mathrm{D}$ information. For radio broadcasting, this problem is increased as listeners cannot see the position and direction of the ball and just rely on the dictations of a commentator. For this situation, more accurate directional information about the ball could help viewers and listeners judge its path.

This paper presents a vibrotactile display for an immersive live sports experience and an overall system concept including tactile data in broadcasting. A vibrotactile display can be easily used to detect the motion of specific object [3], [4], [5]. The prior work allows us to test the feasibility of using a tactile display for sensory compensation in a soccer game scenario in a prototype system. In addition, we propose a methodology for path display that assists a user in recognizing directional movements.

This paper is organized as follows: Section 2 describes the system configuration, and the mapping between the vibrotactile device and the goal area. Section 3 presents a preliminary user study and results. Finally, Section 4 draws some conclusions, and highlights opportunities for future work. 


\section{System Configuration and Mapping Rule}

The system configuration is shown in Fig 1(a). As this is a feasibility study (and for simplicity) we used a virtual, rather than a real, soccer game for our experiment. The system is divided into two parts: a desktop computer and a tactile device. The desktop computer calculates the movement of the virtual ball and transmits the tactile data to the vibrotactile device through Bluetooth to control the vibrating motors sequentially. The vibrotactile device has its own micro controller [6] which controls the vibrators.

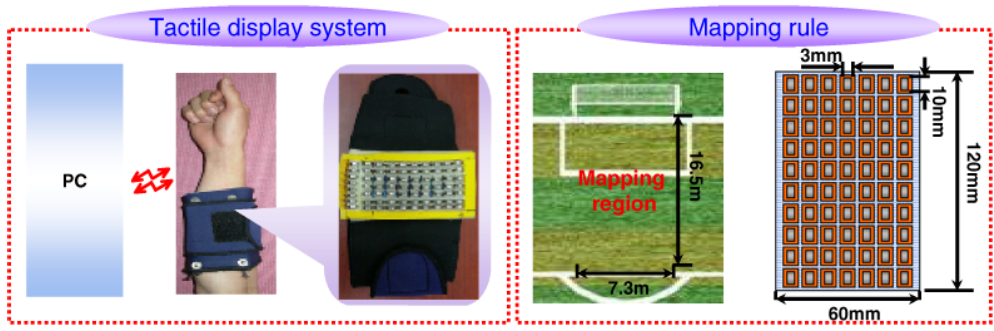

(a)

(b)

Fig. 1. System configuration

As is shown in Fig 1(b), the tactile array is composed of a $(7 \times 10)$ array and attached on the forearm in order to display directional movement of the ball. As they are relatively ergonomic and comfortable for the viewer, the coin type pager motors widely in use cell phones were used in our hardware. They have the additional qualities of being small, light, cheap and low power.

Due to the limited resolution of the tactile display, it was important to carefully select the region of the soccer pitch that would correspond to the device. Since the most interesting event of a soccer game is a goal, the front of the goal (shown in Fig 1(b)) was chosen for the actuator mapping area. We chose a region with a width of 7.3 meters and a length of 16.5 meters. The vibrotactile array is 60 millimeters by 120 millimeters. Therefore, each vibrator represents 1.05 meters width and 1.65 meter length.

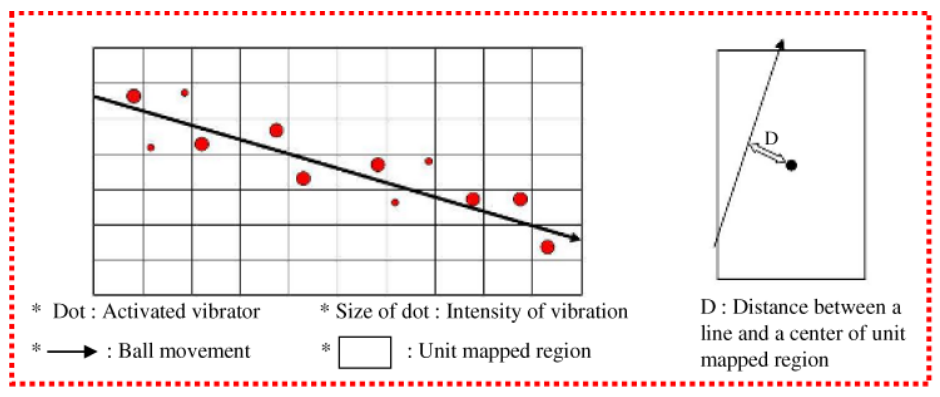

Fig. 2. Control rule of vibrotactile actuator 
The vibrating motors are controlled sequentially to display the movement of a soccer ball on the ground. After some user studies, we found that a "path display" to give correct directional information on the forearm can be effectively achieved by "tracing mode", in which two vibrators along the ball motion trajectory are excited simultaneously with different intensities that are proportional to the distance from the center of the mapped region to the virtual ball position (See Fig. 2). The tracing mode, therefore displays a flow of vibration. Note that each motor is controlled by a PWM (Pulse Width Modulation) signal with duty ratio from $10 \%$ to $90 \%$ for generating different intensities.

\section{User Study and Results}

Two recognition rates have been investigated through preliminary user studies. Firstly, path recognition where we determine at what resolution viewers recognizes the directional movement of a ball with the proposed vibrotactile array. Secondly, goal recognition where we examine how effective the tracing mode is at allowing users to distinguish between goals and near misses. A total 10 subjects were involved in the user studies.

\subsection{Path Recognition}

For path recognition, each subject was trained with six established patterns (Fig 3(a)), experiencing each pattern 3 times. They were then exposed to the patterns again and asked to identify which they were experiencing. The patterns were randomly displayed at eighteen times. Since the vibrotactile device has spatially limited resolution, patterns were established at intervals of five degrees from each other in order to display distinguishable paths. Both tracing and non-tracing mode were tested. The user studies show that although it is difficult for some participants to discriminate adjacent path, most participants could discriminate the directions of a ball in Fig 3(a). While path recognition rate without the tracing mode reached around $81 \%$ mean, the tracing mode shows about $4 \%$ higher recognition rate than the non-tracing mode. In addition, most participants said that they could feel the flow of vibration as if someone scanned their forearm with a fingertip.

\subsection{Goal Recognition}

For goal recognition, cases of the confusing ball motion are established by the trajectories shown in Fig 3(c). When a subject sees a ball motion from the perspective

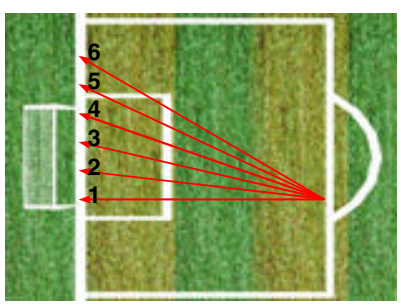

(a)

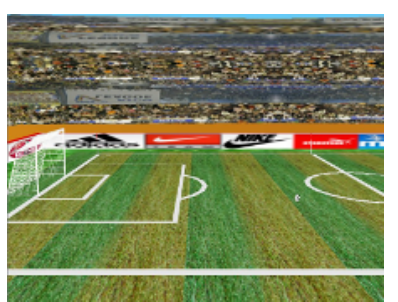

(b)

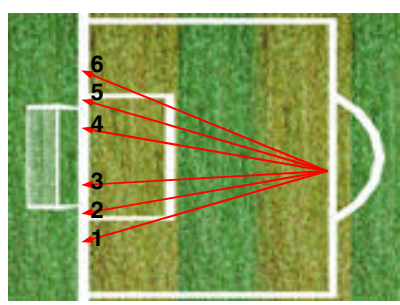

(c)

Fig. 3. User study environments 
shown in Fig 3(b), the simulated 3D soccer playing ground, if can be challenging to judge whether the ball is heading towards the goal or not. Each subject was asked to make this decision in trials with and without the vibrotactile device. Without tactile compensation, accuracy was around $60 \%$ mean. In particular, most subjects had difficulty with cases 2 and 3 . However recognition rate with tactile compensation increased by $33 \%$ (to $80 \%$ accuracy), indicating the tactile cues substantially disambiguated this situation.

\section{Conclusions and Future Works}

We designed a prototype vibrotactile display system to compensate for visually confusing movements of a ball in a soccer match. We also proposed a tactile display method termed tracing mode that accurately displays the directional information of a moving ball. By displaying a seemingly true ball path on the 2D vibrotactile display plane that maps to the front area of the goal in a soccer pitch, subjects could feel the correct ball paths with an accuracy rate of about $80 \%$. This shows the feasibility of the proposed system. The next phase of this work is to display the 3D movement of a ball in two vibrotactile display planes and to conduct a more comprehensive human factors study to inform the design of future generations of device (perhaps including vertical as well as horizontal 3D compensation). Furthermore, a statistical analysis of the user's responses will be performed. In the long run, the proposed system will be extended to a realistic broadcasting system with image processing, editing, and networking.

\section{Acknowledgment}

This work was supported by the Ministry of Information and Communication through the Realistic Broadcasting IT Research Center at GIST and by the next generation PC project.

\section{References}

1. S. O'Modhrain and I. Oakley, "TouchTV: Adding Feeling to Broadcast media", Proc. the $1^{\text {st }}$ European Conf. Interactive Television: from Viewer to Actors, pp.41-47, 2003.

2. J. Cha, J. Ryu, S. Kim, S. Eom, and B. Ahn, "Haptic Interaction in Realistic Multimedia Broadcasting", Proc. 5th Pacific Rim Conf. Multimedia on Advances in Multimedia Information Processing, Part III, pp.482-490, 2004.

3. H.Z. Tan and A. Pentland, "Tactual Display for Wearable Computing", Int, Sympo, Wearable Computers, pp.84-89, 1997.

4. Y. Yanagida, M. Kakia, R.W. Lindeman, Y. Kume, and N. Tetsutani, "Vibrotactile Letter Reading using a Low-Resolution Tactor Array”, Proc. of the 12th Symp. on Haptic Interfaces for Virtual Environment and Teleoperator Systems, pp.400-406, 2004.

5. U. Yang, Y, Jang, and G.J. Kim, "Designing a Vibro-Tactile Wear for "Colse Range" Interaction for VR-based Motion Training", Proc. 12th International Conference on Artificial Reality and Telexistence(ICAT2002), pp.4-9, 2002.

6. http://www.atmel.com/dyn/resources/prod_documents/doc2467.pdf 